Kamyar M. Davoudi*, Hossein M. Davoudi and Elias C. Aifantis

\title{
Nanomechanics of a screw dislocation in a functionally graded material using the theory of gradient elasticity
}

\begin{abstract}
The modest aim of this short article is to provide some new results for a screw dislocation in a functionally graded material within the theory of gradient elasticity. These results, based on a displacement formulation and the Fourier transform technique, complete earlier findings obtained with the stress function method and extends them to the case of the second strain gradient elasticity. Rigorous and easy-to-use analytical expressions for the displacements, strains, and stresses are obtained, which are free from singularities at the dislocation line.
\end{abstract}

Keywords: functionally graded material; screw dislocation; second strain gradient elasticity.

*Corresponding author: Kamyar M. Davoudi: School of Engineering and Applied Sciences, Harvard University, Cambridge, MA 02138 , USA, e-mail: davoudi@seas.harvard.edu

Hossein M. Davoudi: Department of Mathematics, Tarbiat Moallem University, Tehran, Iran

Elias C. Aifantis: Center for the Mechanics of Material Instabilities, and Manufacturing Processes (MMIMP), Michigan Technological University, Houghton, MI 49931, USA; and Laboratory of Mechanics and Materials (LMM), Polytechnic School, Aristotle University of Thessaloniki, P.O. Box 468, Thessaloniki 54124, Greece

\section{Introduction}

In an earlier article published a few years ago [1], the stress function technique was used to derive stress and strain fields for a screw dislocation in a functionally graded material by using the theory of first gradient elasticity. Analytical non-singular expressions were derived for the strains and the (first-order) stresses; however, the double stresses remained singular. Such results are derived here by using the Fourier transform technique, which, in addition, provides exact analytical expressions for the displacement field. Moreover, the problem is reconsidered within the framework of "second strain gradient elasticity," which eliminates the singularities from the double stress expressions as well. Recent work on gradient elasticity $[2,3]$ has revealed the need of using higher-order gradients of strain in the stress-strain relation in order to interpret experimental results pertaining to dislocation density tensor and more accurately describe the details of the relevant stress-strain fields near the core of dislocations contained in small volumes. This is the case in particular, for dislocations contained in functionally graded materials (FGMs). Generally, FGMs refer to heterogeneous composite materials, in which mechanical properties are intentionally made to vary smoothly and continuously from point to point. This is controlled by the variation of the volume fraction of the constituent materials. Ceramic/ ceramic and metal/ceramic are typical examples of FGMs $([4,5]$ and references quoted therein). Although several aspects of FGMs have been reviewed comprehensively ([6-8] and references quoted therein), only few investigations have been made to assess the role of the dislocations in FGMs.

With the exception of ref. [1], the classic theory of linear elasticity was routinely used to calculate the elastic fields produced by defects (dislocations and disclinations) in FGMs. However, classic continuum theories are scale invariant in which no intrinsic length appears and so fails when one attempts to explain the nanoscale phenomena near defects. As a result, elastic singularities are present in these solutions and size effects that dominate in small volumes cannot be captured. These undesirable features are removed within the second strain gradient elasticity formulation presented in this article. The solutions obtained herein by using the Fourier transform technique and a displacement formulation are reduced to the corresponding expressions of classic elasticity and first gradient elasticity as, for example, were obtained in ref. [1] through the use of the stress function approach. As a result, analytical expressions for the displacement field (in addition to those for the stresses and strains) are derived. The extra dividend is the derivation of non-singular expressions for the double stresses that diverge near the dislocation core in the first-order strain gradient theory. 


\section{Classic solution}

We consider a screw dislocation with Burgers vector $b=(0$, $0, b_{z}$ ) in an infinite medium with a varying shear modulus $\mu=\mu(y)=\mu_{0} e^{2 a y}(a \geq 0)$ in the framework of classic elasticity. This is a problem of anti-plane shear with the only nonvanishing component of displacement $u_{z}^{0}(x, y)$ satisfying the displacement equilibrium equation

$$
\left(\nabla^{2}-2 a \frac{\partial}{\partial y}\right) u_{z}^{0}=0
$$

where $\nabla^{2}$ denotes the Laplacian. Using the substitution $u_{z}^{0}=w^{0} e^{-a y}$, we obtain

$$
\left(\nabla^{2}-a^{2}\right) w^{0}=0
$$

which by means of the Fourier transform

$$
\tilde{f}(s)=\mathrm{F}\{f(x) ; x \rightarrow s\}=\int_{-\infty}^{\infty} f(x) e^{-i s x} d x
$$

where $i=\sqrt{-1}$, is reduced to the following ordinary differential equation

$$
\left(-s^{2}-a^{2}+\frac{d^{2}}{d y^{2}}\right) \tilde{w}=0
$$

As $u_{z}^{0}$ is finite everywhere, we arrive at

$$
\tilde{u}_{z}^{0}=e^{-a y} \begin{cases}A(s) e^{-y \sqrt{s^{2}+a^{2}}} & (y>0), \\ B(s) e^{y \sqrt{s^{2}+a^{2}}} & (y<0),\end{cases}
$$

where the two unknown functions, $A(s)$ and $B(s)$, are constants with respect to $y$. In view of the present dislocation configuration, we have

$$
\begin{gathered}
u_{z}^{0}\left(x, 0^{+}\right)-u_{z}^{0}\left(x, 0^{-}\right)=b_{z} H(-x) \\
\varepsilon_{z y}^{0}\left(x, 0^{+}\right)=\varepsilon_{z y}^{0}\left(x, 0^{-}\right) \Leftrightarrow \frac{\partial u_{z}^{0}}{\partial y}\left(x, 0^{+}\right)=\frac{\partial u_{z}^{0}}{\partial y}\left(x, 0^{-}\right),
\end{gathered}
$$

where $\varepsilon_{z y}^{0}$ is the classic strain, and $H(-x)$ is the Heaviside step function. Taking the Fourier transform of the above conditions, we have

$$
\tilde{u}_{z}^{0}\left(x, 0^{+}\right)-\tilde{u}_{z}^{0}\left(x, 0^{-}\right)=b_{z}\left(\pi \delta(s)+\frac{i}{s}\right) ; \frac{\partial \tilde{u}_{z}^{0}}{\partial y}\left(s, 0^{+}\right)=\frac{\partial \tilde{u}_{z}^{0}}{\partial y}\left(s, 0^{-}\right),
$$

and, thus, the unknown functions $A(s)$ and $B(s)$ are determined as

$$
A(s)=\frac{-i a}{2 s \sqrt{s^{2}+a^{2}}}+\frac{i}{2 s}, \quad B(s)=\frac{-i a}{2 s \sqrt{s^{2}+a^{2}}}-\frac{i}{2 s}-\pi \delta(s) .
$$

It follows that

$$
\begin{aligned}
\tilde{u}_{z}^{0}=b_{z} e^{-a y} & {\left[\frac{-i a}{2 s \sqrt{s^{2}+a^{2}}} e^{-|y| \sqrt{s^{2}+a^{2}}}+\operatorname{sgn}(y) \frac{i}{2 s} e^{-|y| \sqrt{s^{2}+a^{2}}}\right.} \\
& \left.-\pi \delta(s) H(-y) e^{a y}\right]
\end{aligned}
$$

and by taking the inverse Fourier transform, i.e.,

$$
u_{z}^{0}(x, y)=\mathrm{F}^{-1}\left\{\tilde{u}_{z}^{0}(s, y) ; s \rightarrow x\right\}=\frac{1}{2 \pi} \int_{-\infty}^{\infty} \tilde{u}_{z}^{0}(s, y) e^{i s x} d s
$$

we obtain (in view of the symmetry of the integral) the final expression

$$
\begin{aligned}
u_{z}^{0}= & \frac{b_{z}}{2 \pi} e^{-a y} \int_{0}^{\infty} \frac{a \sin (s x)}{s \sqrt{s^{2}+a^{2}}} e^{-|y| \sqrt{s^{2}+a^{2}}} d s \\
& -\operatorname{sgn}(y) \frac{b_{z}}{2 \pi} e^{-a y} \int_{0}^{\infty} \frac{\sin (s x)}{s} e^{-|y| \sqrt{s^{2}+a^{2}}} d s-\frac{b_{z}}{2} H(-y) .
\end{aligned}
$$

Next, we note that in the small strain theory, the (compatible) total strain $\varepsilon_{i j}^{T}$ may be written as

$$
\varepsilon_{i j}^{T}=(1 / 2)\left(u_{i, j}+u_{j, i}\right)=\varepsilon_{i j}+\varepsilon_{i j}^{P},
$$

where $\varepsilon_{i j}$ and $\varepsilon_{i j}^{P}$ denote the usual (incompatible) elastic and plastic strains, respectively. It follows that

$$
\begin{aligned}
\varepsilon_{z x}^{0 T}= & \frac{1}{2} \frac{\partial u_{z}^{0}}{\partial x}=\frac{b_{z}}{4 \pi} e^{-a y} \int_{0}^{\infty} \frac{a \cos (s x)}{\sqrt{s^{2}+a^{2}}} e^{-|y| \sqrt{s^{2}+a^{2}}} d s \\
& -\operatorname{sgn}(y) \frac{b_{z}}{4 \pi} e^{-a y} \int_{0}^{\infty} \cos (s x) e^{-|y| \sqrt{s^{2}+a^{2}}} d s, \\
\varepsilon_{z y}^{0 T}= & \frac{1}{2} \frac{\partial u_{z}^{0}}{\partial y}=\frac{b_{z}}{4 \pi} e^{-a y} \int_{0}^{\infty} \frac{s \sin (s x)}{\sqrt{s^{2}+a^{2}}} e^{-|y| \sqrt{s^{2}+a^{2}}} d s \\
& -\frac{b_{z}}{2 \pi} \delta(y) \int_{0}^{\infty} \frac{\sin (s x)}{s} d s+\frac{b_{z}}{4} \delta(y) .
\end{aligned}
$$

With the help of the identities

$$
\int_{0}^{\infty} \frac{\sin (s x)}{s} d s=\frac{\pi}{2} \operatorname{sgn}(x), \quad \int_{0}^{\infty} \frac{\cos (s x)}{\sqrt{s^{2}+a^{2}}} e^{-|y| \sqrt{s^{2}+a^{2}}} d s=K_{0}(\text { ar }),
$$

where $r=\sqrt{x^{2}+y^{2}}$ and $K_{n}$ denotes the modified Bessel function of the second kind and of order $n$, the integrals appearing in Eq. (10) can readily be evaluated to give 


$$
\begin{aligned}
& \varepsilon_{z x}^{0 T}=\frac{b_{z}}{4 \pi} e^{-a y}\left[a K_{0}(a r)-\frac{a y}{r} K_{1}(a r)\right], \\
& \varepsilon_{z y}^{0 T}=\frac{b_{z}}{4 \pi} e^{-a y} \frac{a x}{r} K_{1}(a r)+\frac{b_{z}}{2} \delta(y) H(-x) .
\end{aligned}
$$

The last term in Eq. (11), which is singular on the half-plane $y=0$ and $x \leq 0$, corresponds to the plastic strain $\varepsilon_{z y}^{0 P}=b_{z} \delta(y) H(-x) / 2$. The other term on the right-hand side of Eq. (11) ${ }_{2}$ may thus be regarded as the elastic strain. Using the constitutive law, $\sigma_{z i}^{0}=2 \mu \varepsilon_{z i}^{0}(i=x, y)$, the stresses read

$$
\begin{aligned}
& \sigma_{z x}^{0}=\frac{b_{z} \mu_{0}}{2 \pi} e^{a y}\left[a K_{0}(a r)-\frac{a y}{r} K_{1}(a r)\right], \\
& \sigma_{z y}^{0}=\frac{b_{z} \mu_{0}}{2 \pi} e^{a y} \frac{a x}{r} K_{1}(a r),
\end{aligned}
$$

which are the same as those earlier obtained in ref. [1] by the stress function approach, and which are singular at the dislocation line.

\section{Strain gradient elasticity solution}

Within a simplified theory of linearized anisotropic theory of the second strain gradient elasticity proposed in ref. [9] (for a corresponding form of the first strain gradient elasticity and a robust method for the solutions of the corresponding boundary value problems, the reader may consult $[10,11])$, the strain energy density has the form

$$
W=\frac{1}{2} C_{i j k l} \varepsilon_{i j} \varepsilon_{k l}+\frac{1}{2} \ell^{2} C_{i j m n} \varepsilon_{m n, k} \varepsilon_{i j, k}+\frac{1}{2} \ell^{\prime 4} C_{i j m n} \varepsilon_{m n, k l} \varepsilon_{i j, k l},
$$

where $\varepsilon_{i j}$ is the elastic strain tensor, $\ell$ and $\ell^{\prime}$ are the internal lengths, and $C_{i j k l}$ is the stiffness tensor of the form

$$
C_{i j k l}=\lambda(\mathbf{x}) \delta_{i j} \delta_{k l}+\mu(\mathbf{x})\left(\delta_{i k} \delta_{j l}+\delta_{j k} \delta_{i l}\right),
$$

with the Lamé constants $\lambda(\mathbf{x})$ and $\mu(\mathbf{x})$ being given functions of the spatial coordinates. The corresponding expressions for the elastic-like first-order stress $\left(\sigma_{i j}^{E}\right)$ and the higherorder double $\left(\tau_{i j k}\right)$ and triple $\left(\tau_{i j k k}\right)$ stresses are given by

$$
\begin{aligned}
\sigma_{i j}^{E} & :=\frac{\partial W}{\partial \varepsilon_{i j}}=C_{i j k l} \varepsilon_{k l}, \tau_{i j k}:=\frac{\partial W}{\partial \varepsilon_{i j, k}}=\ell^{2} C_{i j m n} \varepsilon_{m n, k}, \\
\tau_{i j k l}: & :=\frac{\partial W}{\partial \varepsilon_{i j, k l}}=\ell^{\prime 4} C_{i j m n} \varepsilon_{m n, k l},
\end{aligned}
$$

while the Cauchy stress $\sigma_{i j}$ (note that in the notation of ref. [1] this was denoted by $\sigma_{i j}^{0}$ and termed total stress) satisfies, in the absence of body forces, the usual equilibrium equation
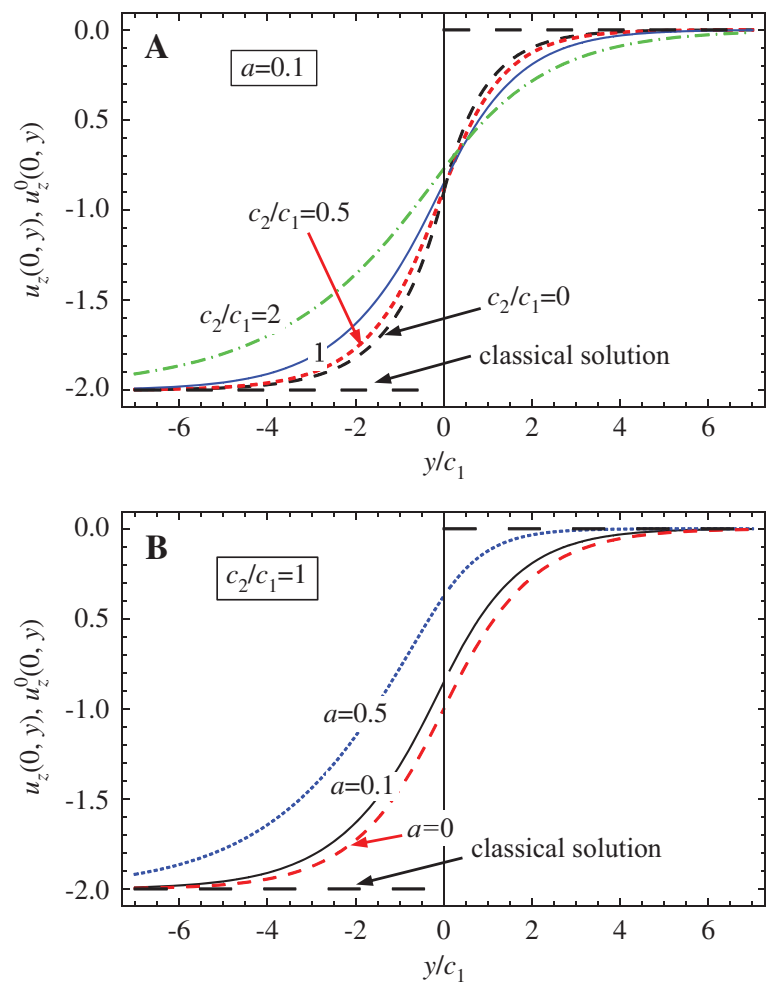

Figure 1 The profile of $u_{z}(0, y)$ in units of $b_{z} / 4$, (A) when $a=0.1$ for $c_{2} / c_{1}=0,0.5,1$, and 2 , and (B) when $c_{2}=c_{1}$ and $a=0,0.1$, and 0.5 . The long-dashed curves in $(A)$ and $(B)$ are pertinent to the classic solutions.

$$
\sigma_{i j, j}=\sigma_{i j, j}^{E}-\tau_{i j k, k j}+\tau_{i j k l, k l j}=0 .
$$

The Cauchy stress, $\sigma_{i j}$, can be identified with the classic stress tensor. For the present case of anti-plane shear, we have

$$
\sigma_{z j}^{E}=2 \mu \varepsilon_{z j}, \quad \tau_{z j k}=2 \ell^{2} \mu \varepsilon_{z j, k}, \quad \tau_{z j k l}=2 \ell^{\prime 4} \mu \varepsilon_{z j, k l} ; \quad(j, k, l=x, y) .
$$

For an exponentially graded material in the $y$-direction, i.e., $\mu=\mu(y)=\mu_{0} e^{2 a y}$, it follows from the above relations that Eq. (14) can be written as

$$
\left[1-c_{1}^{2}\left(\nabla^{2}-2 a \frac{\partial}{\partial y}\right)\right]\left[1-c_{2}^{2}\left(\nabla^{2}-2 a \frac{\partial}{\partial y}\right)\right] \sigma_{z j}^{E}=\sigma_{z j}, \quad(j=x, y)
$$

where $c_{1}^{2}+c_{2}^{2}=\ell^{2}$ and $c_{1}^{2} c_{2}^{2}=\ell^{\prime 4}$. By expressing the stresses in terms of the displacement field in both sides, we have

$$
\begin{aligned}
& {\left[1-c_{1}^{2}\left(\nabla^{2}-2 a \frac{\partial}{\partial y}\right)\right]\left[1-c_{2}^{2}\left(\nabla^{2}-2 a \frac{\partial}{\partial y}\right)\right]\left(\frac{1}{2} \frac{\partial u_{z}}{\partial x_{j}}+\varepsilon_{z j}^{P}\right)} \\
& \quad=\left(\frac{1}{2} \frac{\partial u_{z}^{0}}{\partial x_{j}}+\varepsilon_{z j}^{O P}\right),
\end{aligned}
$$



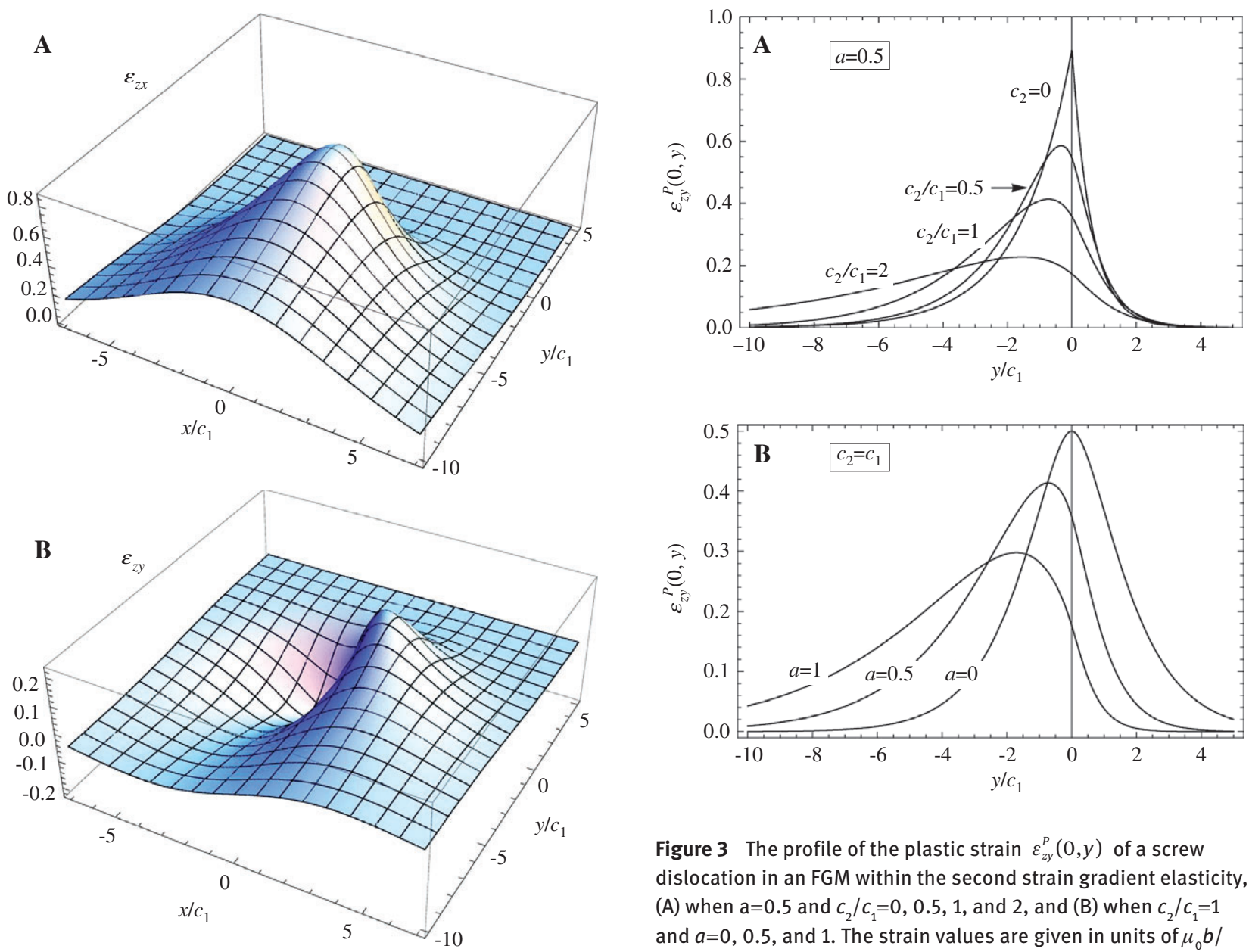

Figure 3 The profile of the plastic strain $\varepsilon_{z y}^{P}(0, y)$ of a screw dislocation in an FGM within the second strain gradient elasticity, (A) when $\mathrm{a}=0.5$ and $c_{2} / c_{1}=0,0.5,1$, and 2 , and (B) when $c_{2} / c_{1}=1$ and $a=0,0.5$, and 1 . The strain values are given in units of $\mu_{0} b /$ $\left(4 \pi c_{1}\right)$.

Figure 2 The elastic strain components, (A) $\varepsilon_{z x}$ and (B) $\varepsilon_{z y}$, of a screw dislocation in an FGM when $a=0.5$ and $c_{2} / c_{1}=0.5$ within the second strain gradient elasticity. The strain values are given in units of $b /$ $\left(4 \pi c_{1}\right)$.

$$
\left[1-c_{1}^{2}\left(\nabla^{2}-a^{2}\right)\right]\left[1-c_{2}^{2}\left(\nabla^{2}-a^{2}\right)\right] w=w^{0}
$$

where $w^{0}$ is given in Section 2. Use of the two-dimensional where $u_{z}$ and $u_{z}^{0}$ are, respectively, the displacement components calculated for gradient and classic elasticity, while $\varepsilon_{z j}^{P}$ and $\varepsilon_{z j}^{O P}$ denote the gradient and classic plastic strains. If we assume that the relation

$$
\left[1-c_{1}^{2}\left(\nabla^{2}-2 a \frac{\partial}{\partial y}\right)\right]\left[1-c_{2}^{2}\left(\nabla^{2}-2 a \frac{\partial}{\partial y}\right)\right] \varepsilon_{z j}^{P}=\varepsilon_{z j}^{O P},
$$

is fulfilled, we will immediately find that the displacement satisfies the following governing differential equation:

$$
\left[1-c_{1}^{2}\left(\nabla^{2}-2 a \frac{\partial}{\partial y}\right)\right]\left[1-c_{2}^{2}\left(\nabla^{2}-2 a \frac{\partial}{\partial y}\right)\right] u_{z}=u_{z}^{0}
$$

As before, by the substitution $u_{z}=w e^{-a y}$, we obtain

$$
\left[1+c_{1}^{2}\left(s^{2}+t^{2}+a^{2}\right)\right]\left[1+c_{2}^{2}\left(s^{2}+t^{2}+a^{2}\right)\right] \tilde{w}=\tilde{\tilde{w}}^{0},
$$

where

$$
\tilde{\tilde{w}}=\mathrm{F}\{\mathrm{F}\{w ; x \rightarrow s\} ; y \rightarrow t\}=\int_{-\infty}^{\infty} \int_{-\infty}^{\infty} w(x, y) e^{-i(s x+t y)} d s d t
$$

and

$$
\begin{aligned}
& \tilde{\tilde{w}}^{0}=\mathrm{F}\left\{\tilde{w}^{0} ; y \rightarrow t\right\}=\frac{-i a}{s\left(\omega^{2}+a^{2}\right)}+\frac{t}{s\left(\omega^{2}+a^{2}\right)}-\frac{\pi \delta(s)}{a-i t} ; \\
& \omega^{2}=s^{2}+t^{2} .
\end{aligned}
$$

Then, the inverse Fourier transform gives 


$$
\begin{aligned}
w & =\frac{1}{(2 \pi)^{2}} \int_{-\infty}^{\infty}\left\{\left(\frac{-i a}{s}+\frac{t}{s}\right) \frac{e^{i(s x+t y)}}{\omega^{2}+a^{2}}\right\} d s d t \\
& +\frac{1}{(2 \pi)^{2}} \int_{-\infty}^{\infty}\left\{-\frac{c_{1}^{2}}{c_{1}^{2}-c_{2}^{2}} \frac{-i a}{s\left(\omega^{2}+\kappa_{1}^{2}\right)}+\frac{c_{2}^{2}}{c_{1}^{2}-c_{2}^{2}} \frac{-i a}{s\left(\omega^{2}+\kappa^{2}\right)}\right\} e^{i(s x+t y)} d s d t \\
& +\frac{1}{(2 \pi)^{2}} \int_{-\infty}^{\infty}\left\{-\frac{c_{1}^{2}}{c_{1}^{2}-c_{2}^{2}} \frac{t}{s\left(\omega^{2}+\kappa_{1}^{2}\right)}+\frac{c_{2}^{2}}{c_{1}^{2}-c_{2}^{2}} \frac{t}{s\left(\omega^{2}+\kappa_{2}^{2}\right)}\right\} e^{i(s x+t y)} d s d t \\
& -\frac{\pi}{(2 \pi)^{2}} \int_{-\infty}^{\infty}\left\{\frac{i}{i a+t}-\frac{c_{1}^{2}}{c_{1}^{2}-c_{2}^{2}} \frac{a+i t}{t^{2}+\kappa_{1}^{2}}+\frac{c_{2}^{2}}{c_{1}^{2}-c_{2}^{2}} \frac{a+i t}{t^{2}+\kappa_{2}^{2}}\right\} e^{i t y} d t,
\end{aligned}
$$

where $\kappa_{j}=\sqrt{a^{2}+1 / c_{j}^{2}}$. If we integrate out the variable $s$ and use the integral relations

$$
\begin{gathered}
\frac{1}{2 \pi} \int_{-\infty}^{\infty} \frac{e^{i s x}}{s\left(s^{2}+k^{2}\right)} d s=\frac{i}{2 k^{2}}\left(1-e^{-|k x|}\right) \operatorname{sgn}(x), \\
\int_{0}^{\infty} \frac{t \sin (t y)}{t^{2}+k^{2}} d t=\frac{\pi}{2} \operatorname{sgn}(y) e^{-|k y|}, \\
\int_{0}^{\infty} \frac{\cos (t y)}{t^{2}+k^{2}} d t=\frac{\pi}{2|k|} e^{-|k y|}
\end{gathered}
$$
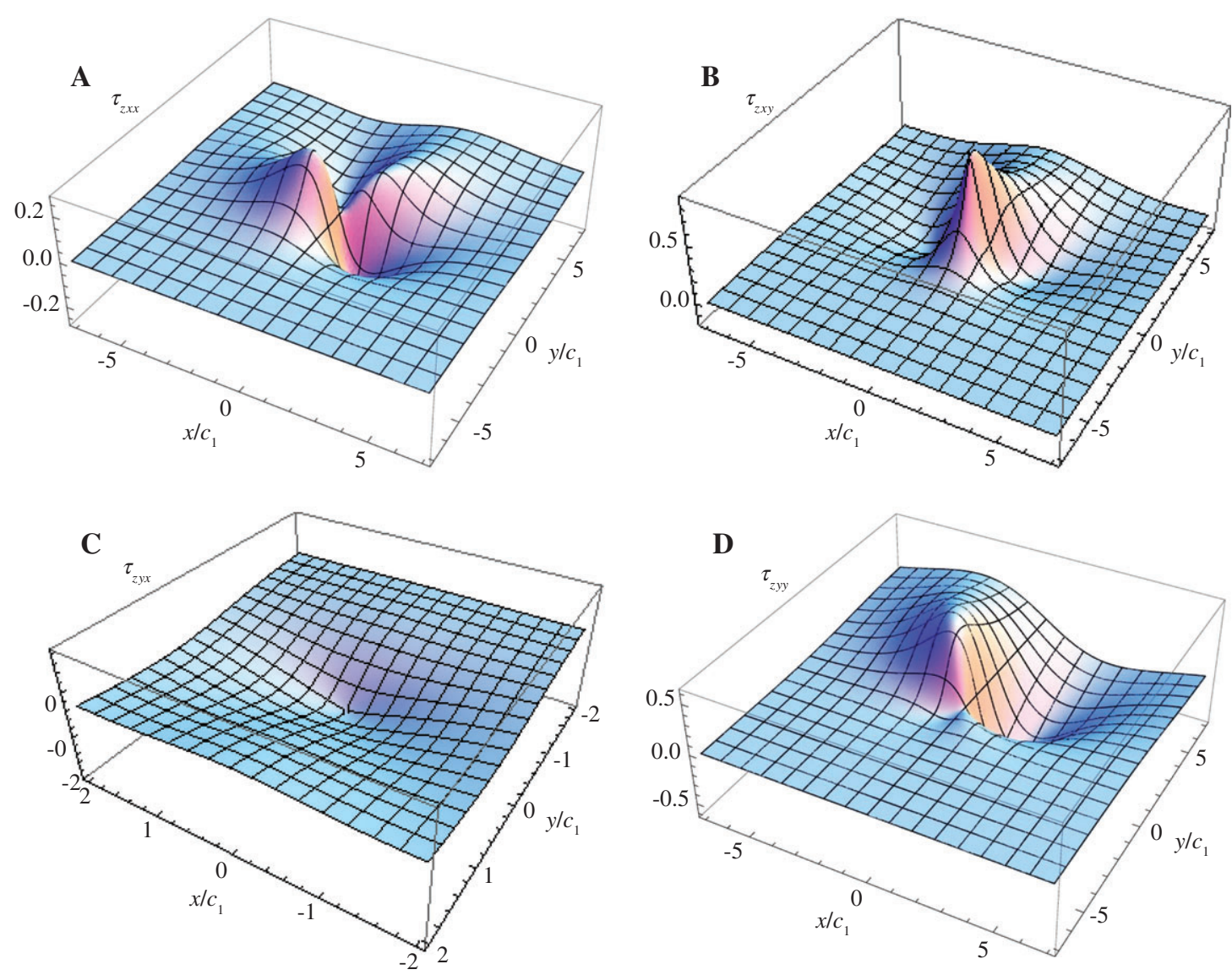

as well as the symmetry properties of these integrals, $u_{z}$ can finally be expressed in terms of sine and cosine integrals as follows

$$
\begin{aligned}
u_{z}= & u_{z}^{0}-\frac{c_{1}^{2}}{c_{1}^{2}-c_{2}^{2}} \frac{b_{z} e^{-a y}}{2 \pi} \int_{0}^{\infty} \frac{t \sin (t y)}{t^{2}+\kappa_{1}^{2}}\left[\operatorname{sgn}(x) e^{-\sqrt{t^{2}+\kappa_{1}^{2}}|x|}+2 H(-x)\right] d t \\
& +\frac{c_{2}^{2}}{c_{1}^{2}-c_{2}^{2}} \frac{b_{z} e^{-a y}}{2 \pi} \int_{0}^{\infty} \frac{t \sin (t y)}{t^{2}+\kappa_{2}^{2}}\left[\operatorname{sgn}(x) e^{-\sqrt{t^{2}+\kappa_{2}^{2}}|x|}+2 H(-x)\right] d t \\
& +\frac{c_{1}^{2}}{c_{1}^{2}-c_{2}^{2}} \frac{b_{z} e^{-a y}}{2 \pi} \int_{0}^{\infty} \frac{a \cos (t y)}{t^{2}+\kappa_{1}^{2}}\left[\operatorname{sgn}(x) e^{-\sqrt{t^{2}+\kappa_{1}^{2}}|x|}+2 H(-x)\right] d t \\
& -\frac{c_{2}^{2}}{c_{1}^{2}-c_{2}^{2}} \frac{b_{z} e^{-a y}}{2 \pi} \int_{0}^{\infty} \frac{a \cos (t y)}{t^{2}+\kappa_{2}^{2}}\left[\operatorname{sgn}(x) e^{-\sqrt{t^{2}+\kappa_{2}^{2}}|x|}+2 H(-x)\right] d t,
\end{aligned}
$$

where $u_{z}^{0}$ is the classic solution given by Eq. (9).

It is easily seen that this expression for $a \rightarrow 0$ coincides with earlier results obtained by the third author and coworkers for homogeneous media and amended in ref. [12]. As Mura [13] neglected the last term of Eq. (21) when $a=0$, and Lazar and Maugin [12] used Mura's calculations, their intermediate calculations are inaccurate, while the final solution is precise. It also turns out that for $\ell=0$ and $\ell^{\prime}=0$,

Figure 4 Double stresses of a straight screw dislocation in an FGM within the second strain gradient when $a=0.5$ and $c_{2} / c_{1}=0.5$. Double stresses are given in units of $\mu_{0} b /(4 \pi)$. 

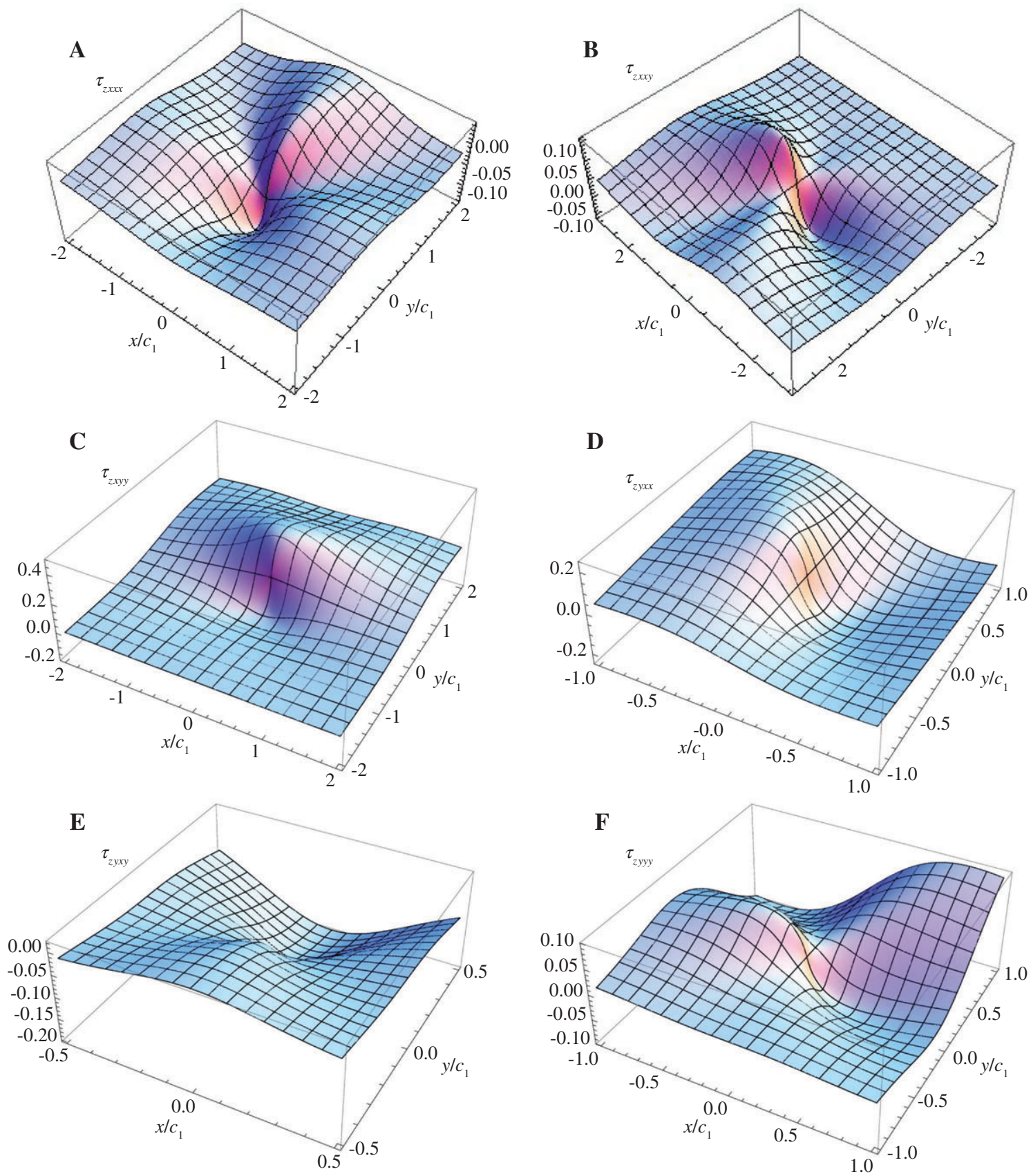

Figure 5 Triple stresses of a straight screw dislocation in an FGM within the second strain gradient when $a=0.5$ and $c_{2} / c_{1}=0.5$. Double stresses are given in units of $\mu_{0} b c_{1} /(4 \pi)$.

the result reduces to Eq. (9). When $x \rightarrow 0$, the displacement field can be expressed in an explicit form

$$
\begin{gathered}
u_{z}(0, y)=\frac{b_{z}}{2} H(-y)+\frac{b_{z}}{4} \frac{e^{-a y}}{c_{1}^{2}-c_{2}^{2}}\left[-c_{1}^{2} \operatorname{sgn}(y) e^{-\kappa_{1}|y|}\right. \\
\left.+c_{2}^{2} \operatorname{sgn}(y) e^{-\kappa_{2}|y|}+c_{1}^{2} \frac{a}{\kappa_{1}} e^{-\kappa_{1}|y|}-c_{2}^{2} \frac{a}{\kappa_{2}} e^{-\kappa_{2}|y|}\right]
\end{gathered}
$$

It is worth noting that the classic displacement $u_{z}^{0}(0, y)$ has an abrupt jump at the dislocation line $y=0$, while the gradient solution of Eq. (24) is smooth there (Figure 1A, B). For a fixed value of $a$, the larger the ratio $c_{2} / c_{1}$ is, the smoother the solution becomes (Figure 1A). The case of $c_{2}=0$ represents the first gradient solution. The gradient solution tends to the classic displacement when $y \rightarrow \pm \infty$, as expected. 

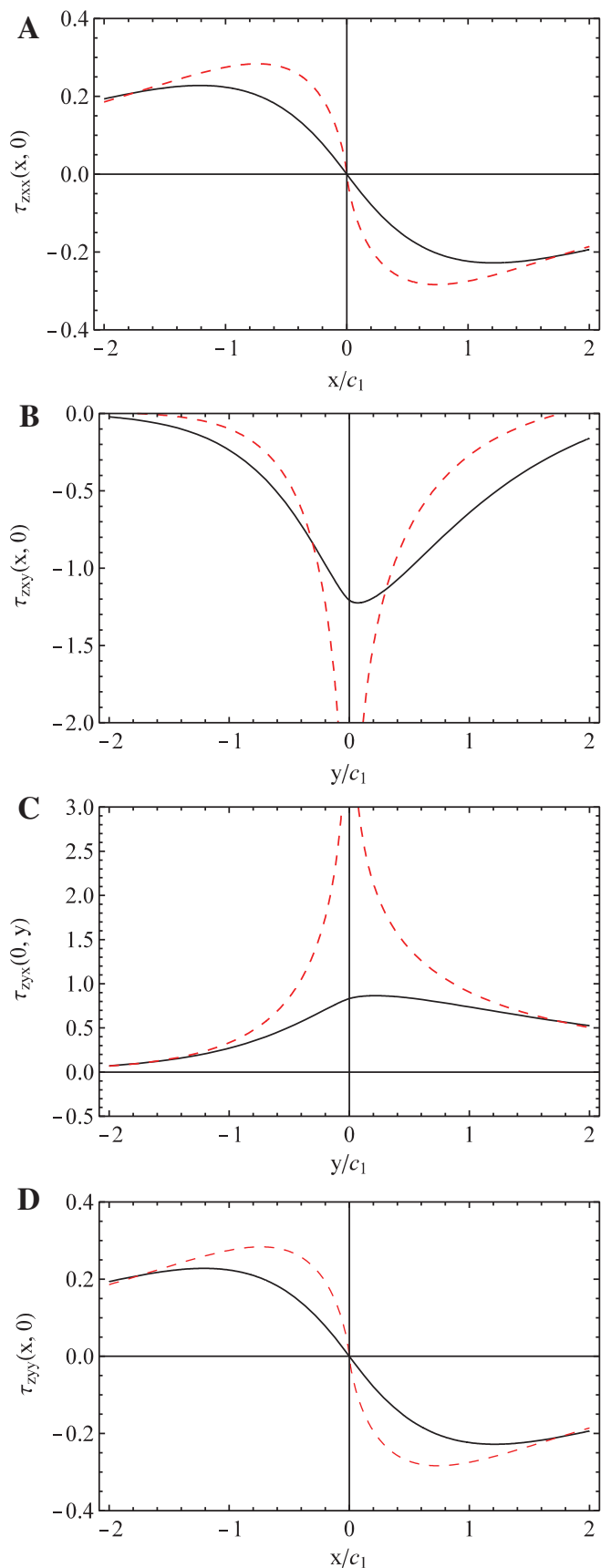

Figure 6 Comparison between double stresses within the first strain gradient (dashed lines) and the second strain gradient (solid lines). It is apparent that $\tau_{z x y}$ and $\tau_{z y x}$ are singular in the first strain gradient.

Using the definition of strain field, the total strains read

$$
\begin{aligned}
\varepsilon_{z x}^{T}=\varepsilon_{z x}^{0}+\frac{b_{z}}{4 \pi} \frac{e^{-a y}}{c_{1}^{2}-c_{2}^{2}} & \\
& {\left[-c_{1}^{2} a K_{0}\left(\kappa_{1} r\right)+c_{2}^{2} a K_{0}\left(\kappa_{2} r\right)+c_{1}^{2} \frac{\kappa_{1} y}{r} K_{1}\left(\kappa_{1} r\right)-c_{2}^{2} \frac{\kappa_{2} y}{r} K_{1}\left(\kappa_{2} r\right)\right], }
\end{aligned}
$$

$$
\begin{aligned}
\varepsilon_{z y}^{T}= & \varepsilon_{z y}^{0}+\frac{b_{z}}{4 \pi} \frac{e^{-a y}}{c_{1}^{2}-c_{2}^{2}} \frac{x}{r}\left[-c_{1}^{2} \kappa_{1} K_{1}\left(\kappa_{1} r\right)+c_{2}^{2} \kappa_{2} K_{1}\left(\kappa_{2} r\right)\right] \\
& +\frac{b_{z}}{4 \pi} \frac{e^{-a y} c_{1}^{2}}{c_{1}^{2}-c_{2}^{2}} \int_{0}^{\infty} \frac{\cos (t y)}{1+c_{1}^{2}\left(t^{2}+a^{2}\right)}\left[\operatorname{sgn}(x) e^{-|x| \sqrt{t^{2}+\kappa_{1}^{2}}}+2 H(-x)\right] d t \\
& -\frac{b_{z}}{4 \pi} \frac{e^{-a y} c_{2}^{2}}{c_{1}^{2}-c_{2}^{2}} \int_{0}^{\infty} \frac{\cos (t y)}{1+c_{2}^{2}\left(t^{2}+a^{2}\right)}\left[\operatorname{sgn}(x) e^{-|x| \sqrt{t^{2}+\kappa_{2}^{2}}}+2 H(-x)\right] d t,
\end{aligned}
$$

where $\varepsilon_{z x}^{0}$ and $\varepsilon_{z y}^{0}$ are the classic (elastic) strains given in Section 2. It is seen that $\varepsilon_{z x}^{T}$ does not contain a plastic part, while $\varepsilon_{z y}^{T}$ is decomposed into the elastic and plastic strains (Figure 2), i.e., $\varepsilon_{z x}=\varepsilon_{z x}^{T} ; \varepsilon_{z x}^{P}=0$, and

$\varepsilon_{z y}=\varepsilon_{z y}^{0}+\frac{b_{z}}{4 \pi} \frac{e^{-a y}}{c_{1}^{2}-c_{2}^{2}} \frac{x}{r}\left[-c_{1}^{2} \kappa_{1} K_{1}\left(\kappa_{1} r\right)+c_{2}^{2} \kappa_{2} K_{1}\left(\kappa_{2} r\right)\right], \varepsilon_{z y}^{P}=\varepsilon_{z y}^{T}-\varepsilon_{z y}$.

It is also follows that the expression for the plastic strain, $\varepsilon_{z y}^{P}(0, y)$, is given by the simple formula

$$
\varepsilon_{z y}^{p}(0, y)=\frac{b_{z}}{8} \frac{e^{-a y}}{c_{1}^{2}-c_{2}^{2}}\left[\frac{e^{-\kappa_{1}|y|}}{\kappa_{1}}-\frac{e^{-\kappa_{2}|y|}}{\kappa_{2}}\right] .
$$

The maximum value of $\varepsilon_{z y}^{P}(0, y)$ decreases as $c_{2} / c_{1}$ or $a$ increases (Figure 3).

The lower-order elastic-like stresses are given by the following expressions:

$$
\begin{aligned}
\sigma_{z x}^{E}= & \sigma_{z x}^{0}+\frac{b_{z} \mu_{0} e^{a y}}{2 \pi\left(c_{1}^{2}-c_{2}^{2}\right)}\left[-c_{1}^{2} a K_{0}\left(\kappa_{1} r\right)+c_{2}^{2} a K_{0}\left(\kappa_{2} r\right)\right. \\
& \left.+c_{1}^{2} \frac{\kappa_{1} y}{r} K_{1}\left(\kappa_{1} r\right)-c_{2}^{2} \frac{\kappa_{2} y}{r} K_{1}\left(\kappa_{2} r\right)\right] \\
\sigma_{z y}^{E}= & \sigma_{z y}^{0}+\frac{b_{z} \mu_{0} e^{a y}}{2 \pi\left(c_{1}^{2}-c_{2}^{2}\right)} \frac{x}{r}\left[-c_{1}^{2} \kappa_{1} K_{1}\left(\kappa_{1} r\right)+c_{2}^{2} \kappa_{2} K_{1}\left(\kappa_{2} r\right)\right],
\end{aligned}
$$

where $\sigma_{z x}^{0}$ and $\sigma_{z y}^{0}$ are the classic stresses given by Eq. (12). It is seen from the above expressions that $\sigma_{z x}^{E}$ is still symmetric with respect to plane $x=0$, while $\sigma_{z y}^{E}$ has lost symmetry with respect to plane $y=0$. Higher-order stresses, $\tau_{i j k}$ and $\tau_{i j k \text { p }}$ can be calculated easily using the fact that

$$
\frac{d}{d z} K_{n}(z)=\frac{n}{2} K_{n}(z)-K_{n+1}(z) .
$$

Figures 4 and 5 show how double stresses and triple stresses vary, respectively, when $c_{2} / c_{1}=0.5$. It is worth mentioning that within the second strain gradient theory, $\tau_{(z x) x} \neq-\tau_{(z y) y}$ in contrast to homogeneous medium. Because

$$
\begin{gathered}
K_{0}(z) \sim-\log (z / 2), \text { as } z \rightarrow 0, \\
K_{n}(z) \sim \frac{1}{2}(n-1) !\left(\frac{z}{2}\right)^{-n}, \text { as } z \rightarrow 0,
\end{gathered}
$$


it can be easily shown that neither double stresses nor triple stresses are singular anymore within the second strain gradient theory, in contrast to the first strain gradient in which some components of the double stress field remain singular (Figure 6). More details, and for this problem, their physical implications to possible improvements of designing FGMs and the expressions for higher stresses, will be given in a forthcoming publication.

\section{Conclusions}

In this work, a screw dislocation in a material exponentially graded in one direction is studied. The displacement field approach and the Fourier transform technique are used to find the displacement and strain fields, and consequently the stress field, in the framework of classic and strain gradient theories. While the classic displacement field has a discontinuity on an arbitrary branch-cut, the displacement field within the gradient theory is smooth everywhere. Accordingly, strain and stress fields in the classic continuum theory, which are singular at the dislocation line, become regular in the gradient theory. In the framework of the first strain gradient, some double stresses are singular at the dislocation line; however, within the second strain gradient theory, not only all double stresses but also triple stresses are regular, similar to the case of a homogeneous medium. When $a$ or $c_{2} / c_{1}$ increases, the fields become smoother.

\section{References}

[1] Lazar M. Mech. Res. Commun. 2007, 34, 305-311.

[2] Kioseoglou J, Dimitrakopulos GP, Komninou Ph, Karakostas Th, Aifantis EC. J. Phys. D: Appl. Phys. 2008, 41, 035408.

[3] Kioseoglou J, Konstantopoulos I, Ribarik G, Dimitrakopulos GP, Aifantis EC. Microsyst. Techol. 2009, 15, 117-121.

[4] Chan Y, Paulino GH, Fannjiang AC. J. Appl. Mech. 2008, 75, 061015.

[5] Paulino GH, Fannjiang AC, Chan Y-S. J. Appl. Mech. 2003, 70, 531-542.

[6] Erdogan F. Compos. Eng. 1995, 5, 753-770.

[7] Markworth AJ, Ramesh KS, Parks WP. J. Mater. Sci. 1995, 30, 2183-2193.

[8] Suresh S, Mortensen A. Fundamentals of Functionally Graded Materials. London: ASM International and the Institute of Materials, IOM Communications Ltd., 1998.

[9] Lazar M, Maugin GA, Aifantis EC. Int. J. Solids Struct. 2006, 43, 1787-1817.

[10] Ru CQ, Aifantis EC. Acta Mech. 1993, 101, 59-68.

[11] Aifantis EC. Mech. Mater. 2003, 35, 259-280.

[12] Lazar M, Maugin GA. Proc. R. Soc. A 2006, 462, 3465-3480.

[13] Mura T. Micromechanics of Defects in Solids, 2nd ed., Dordrecht, the Netherlands: Martines, Nijhoff Publishers, 1982. 